\title{
Stellar population study in early-type galaxies: an approach from the $\mathrm{K}$ band
}

\author{
Esther Mármol-Queraltó ${ }^{1}$, Nicolás Cardiel ${ }^{1}$ P. Sánchez-Blázquez ${ }^{2}$, \\ S.C. Trager ${ }^{3}$, R.F. Peletier ${ }^{3}$, H. Kuntschner ${ }^{4}$, D.R. Silva ${ }^{5}$, \\ A.J. Cenarro ${ }^{2}$, A. Vazdekis ${ }^{2} \&$ J. Gorgas ${ }^{1}$ \\ ${ }^{1}$ Dept. of Astrofísica, Universidad Complutense de Madrid, Ciudad Universitaria, E28040, \\ Madrid, Spain email: emq@astrax.fis.ucm.es \\ ${ }^{2}$ Instituto de Astrofísica de Canarias. c/ Vía Láctea S/N, E38205, La Laguna, Tenerife, Spain \\ ${ }^{3}$ Kapteyn Astronomical Institute, University of Groningen, Postbus 800,9700 Av Groningen, \\ the Netherlands \\ ${ }^{4}$ Space Telescope - European Coordinating Facility, Karl-Schwarzschild-Str. 2, D-85748 \\ Garching, Germany \\ ${ }^{5}$ National Optical Astronomy Observatory, 950 North Cherry Avenue, Tucson, AZ, 85719 USA
}

\begin{abstract}
A full understanding of the physical properties of integrated stellar systems demands a multiwavelength approach since each spectral window shows us the contribution of different types of stars. However, most of the observational effort in stellar population studies has been focused on the optical range. Now, the new generation of instruments allow us to explore the K band, where RGB and AGB stars dominate the light of the integrated spectra. Here we present a K-band spectroscopic analysis of early-type galaxies in different environments. Our sample comprises 12 field early-type galaxies observed with ISAAC at VLT with medium resolution, and they are compared with 11 Fornax cluster galaxies previously reported by Silva et al. (2008). The clear differences found in the infrared $\mathrm{D}_{\mathrm{CO}}$ and $\mathrm{NaI}$ indices between field and Fornax galaxies are discussed, trying to solve the puzzle formed by the near-infrared and optical measurements.
\end{abstract}

Keywords. galaxies: clusters: general - galaxies: elliptical and lenticular, $\mathrm{cD}$ - galaxies: evolution — galaxies: formation — galaxies: stellar content

\section{Motivation}

The near-infrared (near-IR) window has been poorly exploited due to the lack of the appropriate instrumentation. However, this situation is changing with the availability of more sensitive instruments and detectors, and now we can start facing a multiwavelength study of early-type galaxies, which will help us to understand the complexity of these objects. In particular, the near-IR light is dominated by cool and intrinsically luminous late type stars, specially by supergiants (for populations younger than $50 \mathrm{Myr}$ ), AGB (Asymptotic Giant Branch) stars (for populations up to 1.5-2 Gyr), and early-AGB and tip of the RGB (Red Giant Branch) stars (for old populations). These stars are essential contributors to the near-IR light of intermediate age stellar populations and their contribution to the optical range is hidden by the most important contribution of the turn-off dwarfs and stars at the base of the RGB. Therefore near-IR spectroscopy may allow a breaking of the degeneracy between multiple stellar populations that plagues optical absorption-line strength studies.

The study of galaxies in different environments is a powerful tool to understand their evolution and star formation history, as several works in the optical range have revealed (e.g., Sánchez-Blázquez et al. 2003 -hereafer SB03-, Kuntschner et al. 2002; Thomas 
et al. 2005; Collobert et al. 2006). Up to date, the only detailed spectroscopic study in the $\mathrm{K}$ band of early-type galaxies in different environments was presented by Mobasher \& James. These authors analyzed the strength of the first CO band at $2.3 \mu \mathrm{m}$ as an indicator of the presence of AGB stars and, therefore, of an intermediate-age population in elliptical galaxies. Their initial findings (Mobasher \& James 1996) of a systematic difference between field and cluster galaxies were later contradicted by James \& Mobasher (1999), where the authors concluded that they could not detect clear evidences of an offset in the $\mathrm{CO}$ absorption strength between ellipticals in different environments.

More recently, Silva et al. (2008) (hereafter S08) observed a sample of 11 early-type galaxies in the Fornax cluster, and analyzed several line-strength indices in the $\mathrm{K}$ band. These authors found a strong correlation between $\mathrm{NaI}$ and $\mathrm{CO}$ with velocity dispersion $(\sigma)$ for those galaxies without trace of young or intermediate-age populations, as well as between those indices and the optical metallicity indicator $[\mathrm{MgFe}]^{\prime}$ (Thomas et al. 2003). In addition, they showed that the galaxies with young populations in their sample separate from the global trends.

In this work, we present the study of the near-infrared indices $\mathrm{NaI}$ and $\mathrm{D}_{\mathrm{CO}}$ of 12 field early-type galaxies. These data are analyzed simultaneously with the 11 Fornax cluster galaxies from S08, and in comparison with previous optical data. For a more extensive explanation, we refer the reader to Mármol-Queraltó et al. (2009).

\section{The data}

We have obtained long-slit spectra for 12 early-type galaxies living in low-density environments (hereafter, field galaxies). The galaxies were selected from the samples of Sánchez-Blázquez et al. (2006a) and Kuntschner et al. (2002) and chosen to cover a range in $\sigma$ similar to that spanned by S08's Fornax sample. Previous optical work is available for all galaxies (see SB03, Sánchez-Blázquez et al. 2006a, and S08). The data were obtained with the ISAAC near-IR imaging spectrometer mounted at $8.2 \mathrm{~m} \mathrm{UT1/Antu} \mathrm{telescope}$ at Cerro de Paranal (Chile), with exactly the same instrumental configuration employed by $\mathrm{S} 08$, so that both samples can be readily compared.

After a standard data reduction in the infrared using $\mathrm{R}_{\mathrm{E}} \mathrm{D}_{\mathrm{m}}^{\mathrm{uc}} \mathrm{E}$ (Cardiel 1999), galaxy spectra were extracted within a radius corresponding to a $\frac{1}{8} R_{\text {eff }}$ at the observed position angle for each galaxy, where $R_{\text {eff }}$ is the effective radius. On these spectra, we have measured the $\mathrm{NaI}$ index (defined by Frogel et al. 2001) using the broadening correction computed by $\mathrm{S} 08$, and the $\mathrm{D}_{\mathrm{CO}}$ index (as defined by Mármol-Queraltó et al. 2008) which is almost insensitive to this effect.

\section{Results and discussion}

We present in Figure 1 different line-strength indices against $\sigma$ (used as a "proxy" for global metallicity, left column) and $\mathrm{H} \beta$ (an optical "proxy" for the mean age, right column). There is a clear correlation between the indices Mgb, C4668, NaI, $\mathrm{D}_{\mathrm{CO}}$, and $\sigma$ for the Fornax sample (already indicated by Kuntschner 2000 for the optical indices and S08 for the nIR features), while the relations for the field sample exhibit more scatter. We do not find differences in the Mgb measurements between both galaxy samples. In addition, field galaxies show higher $\mathrm{C} 4668, \mathrm{NaI}$ and $\mathrm{D}_{\mathrm{CO}}$ indices than Fornax galaxies at $\sigma<200 \mathrm{~km} \mathrm{~s}^{-1}$. This behaviour is interpreted as differences in the star formation history between both galaxy samples. We are likely seeing the effect of intermediate-age stellar populations in the field galaxies, which are not present in most of their Fornax 


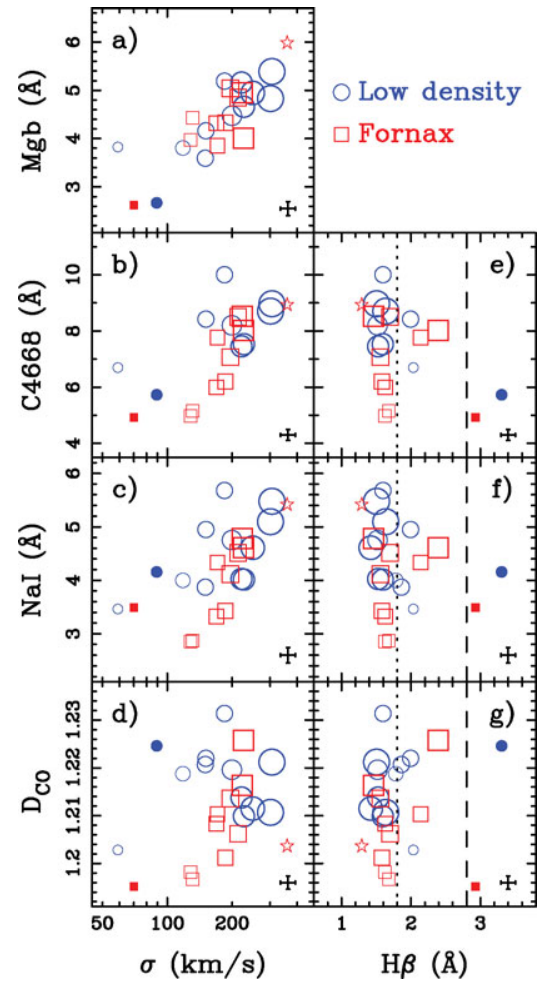

Figure 1. Line-strength indices versus velocity dispersion $\sigma$ (left column) and $\mathrm{H} \beta$ (right column). Blue circles correspond to field galaxies, while red squares represent Fornax cluster galaxies. Star symbol marks cD Fornax galaxy NGC1399. Symbol size increases according to the central velocity dispersion of the galaxies.

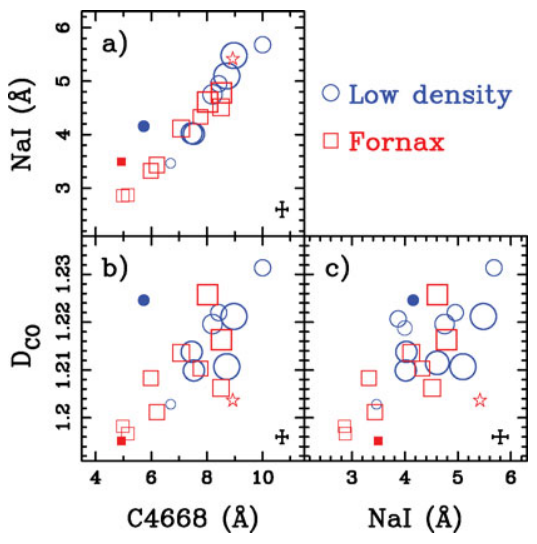

Figure 2. Index-index diagrams for the galaxies in this study. Symbols are explained in Figure 1.

counterparts. Therefore, galaxies living in low-density environments seem to have undergone a more extended star formation history than the Fornax galaxies. In the right column of Fig. 1 we compare different indices with $\mathrm{H} \beta$. As can be seen, we do not find any correlation in this case.

In addition, we notice that, for the field galaxies, the trend of $\mathrm{D}_{\mathrm{CO}}$ with $\sigma$ seems to reverse, with more massive galaxies showing lower values of this index. This could be due to the combined effect of age and metallicity along the $\sigma$ sequence for the field galaxies. If less massive galaxies have a younger mean age (i.e., there is a young population component in them) than more massive ones (e.g. Trager et al. 2000; Thomas et al. 2005; Sánchez-Blázquez et al. 2006b), the index could be larger than for the more massive galaxies, even though the latter would have higher metallicities. This does not happen with the Fornax galaxies as this sample is composed of almost coeval objects (Kuntschner 2000). A more quantitative analysis will have to wait until appropiate models are available. Another explanation was already proposed by Cenarro et al. (2003), who suggested a possible increase of the dwarf-to-giant stars ratio along the mass sequence of elliptical galaxies to explain the anti-correlation found for the CaII triplet features. Under this hypothesis, the massive elliptical galaxies would have a lower fraction of giant (and thus AGB) stars than galaxies with later star formation. Since the $\mathrm{D}_{\mathrm{CO}}$ index decreases when 
the gravity decreases (see Mármol-Queraltó et al. 2008), this scenario could explain the observed anti-correlation. Finally, it is interesting to note that the $\mathrm{cD}$ galaxy of Fornax cluster seem to follow the trend of the field galaxies. This could be explained in this scenario since $\mathrm{cD}$ galaxies are known to have undergone an extended star formation history (e.g. Cardiel et al. 1998), while it is more difficult to fit in the first interpretation.

Finally, we explore possible dependences among the indices $\mathrm{C} 4668, \mathrm{NaI}$ and $\mathrm{D}_{\mathrm{CO}}$ in Fig. 2. There is a extremely tight correlation between NaI and $\mathrm{C} 4666$ indices, interpreted as they are both excellent metallicity indicators for old and intermediate ages. The two galaxies with the highest $\mathrm{H} \beta$ separate from the global trend, as expected due to the sensitivity of these indices to the presence of very young and more metal rich stars. We also find correlations between $\mathrm{D}_{\mathrm{CO}}$ and the indices $\mathrm{C} 4668$ and $\mathrm{NaI}$ (Figs. 2b and 2c), although the scatter is larger in these cases. It can be explained by considering that $\mathrm{D}_{\mathrm{CO}}$ is sensitive to the presence of AGB stars, contrary to the optical C4668 index. In all these diagrams it can also be seen that field galaxies exhibit higher $\mathrm{C} 4668, \mathrm{NaI}$ and $\mathrm{D}_{\mathrm{CO}}$ indices than their Fornax counterparts, as already shown in Fig. 1.

This work has shown the crucial role that the near-IR line-strength indices can play in the understanding of the star formation histories of early-type galaxies. In particular, the $\mathrm{NaI}$ index has revealed to be a metallicity indicator as good as $\mathrm{C} 4668$ in the optical range, while the $\mathrm{D}_{\mathrm{CO}}$ index can be used as tracer of intermediate-age stellar populations. In addition, the differences in $\mathrm{C} 4668, \mathrm{NaI}$, and $\mathrm{D}_{\mathrm{CO}}$, when studying low-mass galaxies in Fornax cluster and in low density environments, can be interpreted as field galaxies having undergone later stellar formation episodes than Fornax galaxies.

\section{References}

Cardiel, N. 1999, Ph.D. Thesis

Cardiel, N., Gorgas, J., \& Aragon-Salamanca, A. 1998, MNRAS, 298, 977

Cenarro, A. J., Gorgas, J., Vazdekis, A., Cardiel, N., \& Peletier, R. F. 2003, MNRAS, 339 , L12

Cenarro, A. J., Sánchez-Blázquez, P., Cardiel, N., \& Gorgas, J. 2004, ApJ (Letters), 614, L101

Collobert, M., Sarzi, M., Davies, R. L., Kuntschner, H., \& Colless, M. 2006, MNRAS, 370,1213

Frogel, J. A., Persson, S. E., Matthews, K., \& Aaronson, M. 1978, ApJ, 220, 75

Frogel, J. A., Stephens, A., Ramírez, S., \& DePoy, D. L. 2001, AJ, 122, 1896

James, P. A. \& Mobasher, B. 1999, MNRAS, 306, 199

Kuntschner, H. 2000, MNRAS, 315, 184

Kuntschner, H., Lucey, J. R., Smith, R. J., Hudson, M. J., \& Davies, R. L. 2001, MNRAS, 323, 615

Kuntschner, H., Smith, R. J., Colless, M., Davies, R. L., Kaldare, R., \& Vazdekis, A. 2002, MNRAS, 337, 172

Mármol-Queraltó, E., Cardiel, N., Cenarro, A. J., Vazdekis, A., Gorgas, J., Pedraz, S., Peletier, R. F., \& Sánchez-Blázquez, P. 2008, A\&SA, 489, 885 (MQ08)

Mármol-Queraltó, E., Cardiel, N., Sánchez-Blázquez, P., Trager, S. C., Peletier, R. F., Kuntschner, H., Silva, D. R., Cenarro, A. J., Vazdekis, A., \& Gorgas, J. 2009, ApJ(Letters), in press (arXiv:0909.5385)

Mobasher, B. \& James, P. A. 1996, MNRAS, 280, 895

Sánchez-Blázquez, P., Gorgas, J., Cardiel, N., Cenarro, J., \& González, J. J. 2003, ApJ (Letters), 590, L91 (SB03)

Sánchez-Blázquez, P., Gorgas, J., Cardiel, N., \& González, J. J. 2006a, A\&SA, 457, 787

-. 2006b, A\&\&A, 457, 809

Silva, D. R., Kuntschner, H., \& Lyubenova, M. 2008, ApJ, 674, 194 (S08)

Thomas, D., Maraston, C., \& Bender, R. 2003, MNRAS, 339, 897

Thomas, D., Maraston, C., Bender, R., \& Mendes de Oliveira, C. 2005, ApJ, 621, 673

Trager, S. C., Faber, S. M., Worthey, G., \& González, J. J. 2000, AJ, 120, 165 